\title{
Performance Modelling and Analysis of Mobile Wireless Networks
}

\author{
Carmen B. Rodríguez-Estrello1, Genaro Hernández Valdez² \\ and Felipe A. Cruz Pérez ${ }^{1}$ \\ Electric Engineering Department, CINVESTAV-IPN ${ }^{1}$ \\ Electronics Department, UAM-A2 \\ Mexico
}

\section{Introduction}

Nowadays, mobile wireless communications are continuously experiencing a growing demand. Therefore, communication systems must be designed and/or enhanced to increase their capacity. In that sense, mathematical analysis must be the first step of designing or improving a network. Thus, powerful mathematical tools which take into account most of the involved parameters in network performance are required to analyze mobile wireless networks.

Wireless networks could be analyzed from link level approach or from system level approach in terms of Quality of Service (QoS). Link level analysis is related to the physical channel characterization and involves statistics such as the probability distributions of the channel states duration, which, in general, are not easily obtained at real cellular networks. In contrast, system level analysis is associated with the characterization of the network's dynamic and involves variables such as channel holding times for successfully and forced terminated calls which are easily obtained at real networks.

QoS in mobile wireless networks means the level of usability and reliability of a network and its services. Consequently, QoS for mobile wireless networks are the basis of dimension and planning. The main concern for an operator is the accessibility and continuity of the connection. As a result, it has been widely accepted that call forced termination probability is one of the most important QoS performance metrics in cellular networks. Forced termination is due to two fundamental features: resource insufficiency and link unreliability. In order to adequately model mobile cellular networks at system level, its mathematical analysis should consider both causes of call forced termination: resource insufficiency and wireless link unreliability. In the literature, resource insufficiency has been widely studied at system level while link unreliability has not been included at system level analysis due to its inclusion entails.

On the other hand, Code Division Multiple Access (CDMA) has been selected as the main multiple access technology of several third generation cellular network standards (Dahlman et al., 2007). CDMA-based cellular systems employ universal frequency reuse factor which makes them interference limited. Consequently, capacity is a direct function of interference 
generated by the users. That is, the number of available radio resources depends on the interference. This special feature is known as "soft capacity". Thus, to correctly asses the performance of a CDMA system it is imperative to consider the effect of interference. The aim of this chapter is to present the mathematical analysis of a CDMA-based system considering link unreliability in a system level analysis, which has been traditionally considered only at link level analysis.

\section{Overview of call forced termination analysis in mobile wireless networks}

One of the most important QoS metrics for the performance evaluation of present and future mobile wireless networks is call forced termination probability. The call forced termination probability is the probability that a call which is not initially blocked be interrupted. In mobile wireless networks, a call is forced to terminate because of two fundamental features: resource insufficiency and link unreliability. Moreover, in the context of packet switched mobile communication networks, call forced termination probability is especially important in the performance evaluation of conversational and real-time services (i.e., voice, audio, music, videophone, videoconference etc.) (Li et al., 2004; Wong et al., 2004).

\subsection{Resource insufficiency}

When a mobile user moves into a different cell during the course of a call, a handoff must be performed. If no radio resources are available in the target cell, the call is said to be forced to terminate due to resource insufficiency. However, in a well-established cellular network and from the call forced termination point of view, handoff failure can be usually a negligible event (Boggia et al., 2005).

\subsection{Link unreliability}

Physical link is said to be unreliable if the experienced signal-to-interference ratio (SIR) is below than a minimum required value (SIR threshold) for more than a specified period of time (time threshold). During the course of a call, the physical link between base station and mobile station may suffer link unreliability due to propagation impairments such as multipath fading, shadowing or path loss, and interference. In CDMA-based systems, link unreliability is experimented due to the initial power allocation. The initial handoff decisions are made for individual connections independent of the other connections or the BS power availability. Therefore, current connections may suffer from link unreliability (Zhao et al., 2006). Hence, the call may be abnormally terminated. In this case, the call is said to be forced to terminate due to link unreliability. In particular, the analysis of measured data traffic supplied by Vodafone Italy is a good example for these phenomena (Boggia et al., 2005). However, only relatively few recent studies have addressed the effect of link unreliability on the performance of mobile wireless communication networks (Elsharabwy \& Le-Ngoc, 2005; Zhang \& Song, 2006; Zhang \& Song, 2005; Zhao et al., 2006; Liu \& Sule, 2004; Naraghi Pour \& Chai, 2006; Kong, 2002).

Most of the work devoted to study the impact of link unreliability on system performance has considered a link level channel model in order to study how the channel impairments affect system performance. Gilbert Elliot and Fritchman channel models have been widely used for this purpose. For instance, authors of (Kong, 2002) proposed a queuing system with 
impaired wireless channel based on the Markov chain approach assuming that the unreliable wireless channel can be modeled by the Gilbert-Elliott channel model. Analysis is performed assuming that each state of the wireless channel is a node. Then, service rate becomes time-varying due to propagation impairments. A model to quantify the performance of a queue with respect to such impaired wireless channel is then developed. In a similar work (Elsharabwy \& Le-Ngoc, 2005), the Gilbert-Elliot channel model is proposed for the downlink performance evaluation of WCDMA cellular systems. In addition, the Gilbert-Elliot channel parameters in terms of the mean fade and non-fade durations are obtained. The proposed QoS performance metrics are based on the satisfieduser criteria recommended by UMTS (i.e., satisfied user probability for speech services and satisfied user probability for data services). Specifically, (Elsharabwy \& Le-Ngoc, 2005), deals with the UMTS QoS recommendation for packet based networks. Authors in (Elsharabwy \& Le-Ngoc, 2005), propose a composite performance index (called satisfieduser probability) based on dropped-call probability and session outage percentage due to link unreliability in wireless communication networks. This performance index is calculated by considering a Gilbert-Elliot channel model with negative exponentially distributed state durations. However, none of the above papers considers users' mobility. Consequently, call forced termination due to resource insufficiency is not addressed.

Other related papers devoted to study the impact of link unreliability on system performance are (Zhang \& Song, 2006), (Zhang \& Song, 2005), which use either the GilbertElliot or Fritchman model to characterize the time-variant wireless channel. Zhang et al. derived mathematical expressions for the probability that a call be successfully completed considering the concurrent impacts of bad quality in the channel and the lack of radio resources. Zhang studied the impact of Rayleigh fast-fading on various teletraffic QoS metrics in wireless networks (i.e., channel holding time, handoff probability, handoff call arrival rate, call blocking probability, call completion probability, and call forced termination probability) taking into account carrier frequency, maximum Doppler frequency and fade margin. From teletraffic point of view, system level-based modeling of link unreliability is preferred over link level-based modeling because fewer state variables are needed. However, mathematical models considered in (Elsharabwy \& Le-Ngoc, 2005), (Zhang \& Song, 2006), (Zhang \& Song, 2005), (Zhao et al., 2006), (Naraghi Pour \& Chai, 2006), (Kong, 2002) are based on link level statistics which are not easily obtained by direct measures. Contrary to the Zhang's works, here, the effect of link unreliability is captured through easily obtained system level quantities which allows including the effect of link unreliability on the channel occupancy directly in the teletraffic analysis.

Only few recently published studies have addressed system level analysis considering link unreliability for wireless networks (Liu \& Sule, 2004; Rodríguez-Estrello et al., 2009). In (Liu \& Sule, 2004), a queuing model to evaluate the performance of CDMA reverse link in a multiple cell scenario was developed. In that work, a quasi-birth-and-death process was used to capture the variation of traffic loads in cells. Then, authors of (Liu \& Sule, 2004), obtained the stationary distribution of the system and some performance indicators, such as the outage probability of existing calls, blocking probability of new calls, average carried traffic in a cell, and dropping frequency of ongoing calls. Nonetheless, neither mobility of users nor soft handoff are modeled in (Liu \& Sule, 2004). Recently, a teletraffic model to evaluate the performance of TDMA/FDMA-based cellular networks considering both resource insufficiency and link unreliability (Rodríguez-Estrello et al., 2009) was proposed. 
The effect of link unreliability is captured by an interruption Poisson process, which is characterized by the mean time of the "unencumbered call interruption time". This interruption process is characterized via system level statistics, based on channel holding time, which is easily measured at base stations (BSs).

\section{System model}

To capture the main features of CDMA-based cellular systems in their performance evaluation, geometrical, users' mobility, interference, and call interruption characteristics are considered.

\subsection{Soft handoff geometrical model}

A homogeneous mobile multi-cellular system with omni-directional antennas located at the center of cells is assumed ${ }^{1}$. Base Stations (BSs) are assumed to use Frequency Division Duplexing (FDD). As in previously published related studies (Zhang \& Lea, 2006; Ma et al., 2006; Hegde \& Sohraby, 2002; Piao et al., 2006; Su et al., 1996 and Kim \& Sung., 1999), we focus on the reverse link as it was found to be the link that limits system performance.

Soft handoff process is performed when a MS receives comparable pilot signal strengths from two or more BSs. At this moment, a communication path is established between the MS and all BSs with comparable pilot signal strengths. Consequently, two or more BSs receive independent streams from the MS. Independent streams can be combined (macro diversity) so that the bit stream is decoded much more reliable than if only one BS were receiving from the MS. When a pilot signal from one BS is considerably stronger than that from the others BSs, the MS is then served by only one BS. Then, soft handoff process can guarantee that the MS is always linked to the BS from which it receives the strongest pilot (Garg, 2000). Here, it is assumed that the MS can communicate with the two nearest BSs only, and, if only propagation path losses are considered ${ }^{2}$, the region where the soft handoff process is performed is the ring area near the borders of the cell. Figure 1 depicts the geometry of the analyzed cells. Ring area is used to represent the area where the soft handoff process is performed.

Thus, an active user in the inner area, referred to as the hard region, is assumed to be connected only to the nearest BS; while mobiles in the outer area, referred to as the soft region, are assumed to be in soft handoff to its two nearest BS's only. As seen in Figure 1, it is clear that the "nominal" coverage of the analyzed cell is increased by the soft regions of the adjacent cells.

The ratio between the area of the hard region to the total cell area (including the overlapped area of the soft regions) is denoted by $p$ while $p^{\prime}$ is the ratio between the area of the hard region to the nominal area of the cell, which is the area of the cell without considering overlapped areas of soft handoff regions.

1 To simplify mathematical analysis, a first approach is to consider the use of omnidirectional antennas. Nonetheless some minor modifications need to be done when directional case is considered.

2 This consideration is acceptable under not severe conditions of shadowing. 


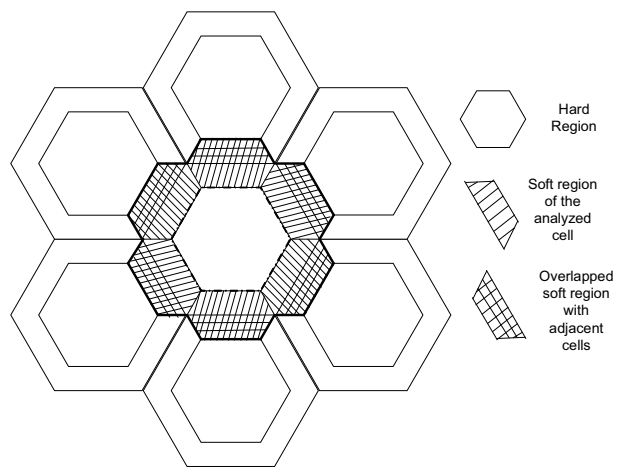

Fig. 1. Network Topology

\subsection{Mobility model}

Macroscopic modeling of mobility in cellular systems is used here. Since the geometrical model previously described considers differentiated coverage regions, users' mobility should be characterized in terms of mean residence times in different coverage zones and the probability $q$ that a user carrying a call originated in the soft region moves to the hard region.

To determine the specific macroscopic statistics, the generalized smooth random mobility model proposed in (Zoonozi \& Dassanayake, 1997) is used here due to its simplicity and versatility to represent several scenarios. The model proposed in (Zoonozi \& Dassanayake, $1997)$ is characterized by the parameter $\alpha$ that limits the range of maximum variation of the current direction of a user, which allows for the representation of different mobility scenarios.

In order, to take into account correlated directions of movement characteristic of smooth random mobility models, probability $q$ takes into consideration the current coverage region as well as the previously visited region (if any) before entering the region under analysis. Hence, three different cases are distinguished in the calculation of $q$ :

1) calls that are originated in the soft region $\left(q_{s}\right)$

2) users that arrived to the soft region of a given cell coming from the hard region of the same cell $\left(q_{s h}\right)^{3}$

3) users that arrived to the soft region of a given cell coming from the soft region of another cell $\left(q_{s s}\right)$.

Residence times in different coverage regions also depend on the correlated moving direction of users. Hence, five different cases are considered:

a) residence time in the soft region when users originate a call in this region $\left(T_{s}\right)$,

\footnotetext{
${ }^{3}$ This probability is zero when a linear movement inside cells is considered, because there is no possibility to return to the previous region. As such, when linear movement is considered, it is not necessary to take into account this probability.
} 
b) residence time in the soft region when users come from a hard region $\left(T_{\text {sh }}\right)$,

c) residence time in the soft region when users come from the soft region of other cell $\left(T_{s s}\right)$,

d) residence time in the hard region when users originate a call in this region $\left(T_{h}\right)$,

e) residence time in the hard region when users come from the soft region $\left(T_{h s}\right)$.

\subsection{Interference model}

Interference power is proportional to the system load and has random characteristics depending on several system variables such as shadowing losses and propagation characteristics. Interference in CDMA systems could be intra-cellular (interference caused by the users which are power controlled by the own-cell) and inter-cellular (caused by the users which are power controlled by another cell).

In this work, as in (Viterbi et al., 1994) interference power is modelled by means of a random variable, $\mathbf{Z}$, which represents the power of the total interference caused by all users in the system.

Since CDMA systems are interference-limited, transmitted power from each mobile user must be controlled to limit interference. However, the received power level should be sufficient to guarantee an adequate energy per bit (Garg, 2000). Then, received power of the $j$-th user should be:

$$
P_{j}=E_{b, j} R
$$

where $E_{b, j}$ is the required energy per bit and $R$ is the transmission rate $(R)$. Normalizing the received power to the product of transmission rate and the maximum acceptable power spectral density of interference $\left(I_{0}\right)$ :

$$
\frac{P_{j}}{I_{0} R}=\frac{E_{b, j} R}{I_{0} R}
$$

Let us define the average required energy per bit normalized to the maximum acceptable power spectral density of interference $\left(I_{0}\right)$ as follows:

$$
\varepsilon_{j}=\frac{E_{b, j}}{I_{0}}
$$

It was shown in (Viterbi et al., 1994) that $\varepsilon_{j}$ could be approximated by a lognormal distribution, that is:

$$
f_{\varepsilon}(\varepsilon)=10^{x / 10}
$$

where $\chi$ is a Gaussian random variable with mean $m_{s}$ and variance $\sigma_{s}$ for users in soft handoff region and $m_{h}$ and $\sigma_{h}$ for users in hard region. Due to macro diversity in soft 
handoff region, users in hard region have different behavior in terms of required average energy per bit. Then, $m_{s}$ and $m_{h}$ and $\sigma_{s}$ and $\sigma_{h}$ are, in general, different.

The transmitted power of each MS is calculated assuming that the effects of fast fading can be ignored 4 . Hence, in order to determine which BS is controlling a MS, only path losses and shadowing are considered. Total attenuation is modeled as the product of the path loss $r^{m}$ by $10^{\zeta / 10}$, which is the log-normal random component representing shadowing losses. Then, for a user at a distance $r$ from a base station, attenuation is proportional to:

$$
a(r, \zeta)=r^{m} 10^{\zeta / 10}
$$

where $\zeta$ is a zero-mean Gaussian random variable.

In the reverse link, there are a significant number of mobiles that contribute to the interference. In accordance with the central limit-theorem, $\mathbf{Z}$ can be approximated by a Gaussian random variable which is completely characterized by its mean and variance.

Due to the soft handoff process, users in hard region have different behavior in terms of interference than users in the soft region. Hence, $\mathbf{Z}$ can be expressed as a sum of four components: intra-cellular and inter-cellular interference caused by the users in both the hard and soft regions.

Then, following the same mathematical procedure described in (Viterbi et al., 1994), the mean and the variance of inter-cell interference can be expressed as a factor of the mean and variance of the intra-cell interference. Let us denote by $f_{h}$ and $f_{s}$ the interference factor for the hard and soft regions, respectively. Thus, the mean and variance of $\mathbf{Z}$ as function of a given number of active users in the hard $(h)$ and soft $(s)$ handoff regions of the cell of interest can be expressed as follows

$$
\begin{gathered}
E[Z(h, s)]=E\left[I_{h}\right]+E\left[I_{s}^{+}\right]+f_{h} E\left[I_{h}\right]+f_{s} E\left[I_{s}^{+}\right] \\
\operatorname{Var}[Z(h, s)]=\operatorname{Var}\left[I_{h}\right]+\operatorname{Var}\left[I_{s}^{+}\right]+f_{h} \operatorname{Var}\left[I_{h}\right]+f_{s} \operatorname{Var}\left[I_{s}^{+}\right]
\end{gathered}
$$

where $E\left[I_{h}\right], E\left[I_{s}^{+}\right], \operatorname{Var}\left[I_{h}\right]$, and $\operatorname{Var}\left[I_{s}^{+}\right]$represent, respectively, mean value and variance of the normalized interference due to the active users in the hard and soft region of the analyzed cell which are power controlled by the analyzed cell. Values of interference factors depend on propagation conditions and are calculated as suggested in (Viterbi et al., 1994). These values are calculated in terms of the number of users in other cells. However, teletraffic analysis of a single cell cannot provide the exact number of users in the whole system and, as a consequence; inter-cell interference should be calculated in terms of the average number of users in neighbouring cells. Let us denote by $\mathbf{H}$ and $\mathbf{S}$ the associated random variables to $h$ and $s$, respectively. Since a homogeneous system is considered and

\footnotetext{
4 The impact of fast fading can be effectively reduced by using channel coding, interleaving, and signal processing techniques such as channel equalization. Additionally, if a spread spectrum transmission technique is employed, the frequency diversity property increases the mitigation effect over fast fading. Consequently, its effects can be ignored (Garg, 2000).
} 
the cells are assumed to be statistically identical, some statistics of the inter-cellular interference power can be calculated considering the average number of active users in the hard region, $E[\mathbf{H}]$, and in the soft region, $E[\mathbf{S}]$, of the cell of interest. Section V shows how $E[\mathbf{H}]$ and $E[\mathbf{S}]$ can be calculated via teletraffic analysis.

\subsection{Proposed interruption model}

Mathematical model used to consider link unreliability is based on the proposed call interruption process in (Rodríguez-Estrello et al., 2009). In (Rodríguez-Estrello et al., 2009), an interruption model and a potential associated time to this process, which is called "unencumbered call interruption time", is proposed. Unencumbered call interruption time is defined as the period of time from the epoch the MS establish a link with a BS until the instant the call would be interrupted due to the wireless link unreliability assuming that the MS has neither successfully completed the call nor has been handed off to another cell. Thus, unencumbered call interruption time depends only on link reliability. Physically, this time represents the period of time in which a call would be terminated under the assumption that both the cell dwell time and the unencumbered service time are infinite. Call interruption time is said to be "unencumbered" because the interruption of a call in progress by link unreliability can or cannot occur, depending on the values of cell dwell time and unencumbered service time.

Unencumbered call interruption time could not be directly measured in real cellular networks, so, it is necessary to relate it with some parameters that could be measured at base stations (i.e., statistics of the unencumbered call service time and the channel holding time for calls forced terminated due to the link unreliability). As a first approach, unencumbered call interruption time is modeled as a negative exponentially distributed random variable (Rodríguez-Estrello et al., 2009).

It is worth mentioning that in CDMA systems, interruption process due to link unreliability of wireless channel in soft region is different from the interruption process due to link unreliability of wireless channel in hard region because of the diversity in the soft handoff region. So that, to differentiate mean unencumbered call interruption time is denoted by $1 / \gamma_{x}$, where the sub index $x$ represents the residence region. Then $x$ could be $\{S, H\}$ for soft and hard regions, respectively.

In order to obtain some statistics, in (Rodríguez-Estrello et al., 2009) is shown that, if unencumbered service time and cell dwell time are considered negative exponentially distributed, $\gamma_{x}$ can be calculated in terms of both channel holding time for calls forced to terminate $\left(1 / \mu_{s d x}\right)$ due to the link unreliability and unencumbered service time $(1 / \mu)$. Thus, $\gamma_{x}$ is given by:

$$
\gamma_{x}=\mu_{s d_{-} x}-\mu
$$

Section 4 shows how the model for taking into account wireless channel unreliability is incorporated in the teletraffic analysis of CDMA-based cellular systems. Then, mathematical expressions for several system level performance metrics are derived. 


\section{Teletraffic analysis}

\subsection{General guidelines}

The general guidelines of the model presented in (Lin et al., 1994) are adopted here to analyze the system under evaluation. The following assumptions have been widely accepted in the literature and allow the analysis presented to be cast in the framework of a multidimensional birth and death processes.

1) A homogeneous multi-cellular system is considered where each cell has associated capacity limits (in terms of the maximum number of simultaneous users in the soft and hard regions of the cell) that determine the valid state space 5 .

2) Only voice service type is considered in the system.

3) New call arrival process follows a Poisson process with mean arrival rate $\lambda_{n}$ per cell considering only the non-overlapped areas. Assuming uniform traffic over the system, the mean values of the new call arrival rate in the hard region is $p^{\prime} \lambda_{n}\left(p^{\prime}\right.$ is considered since it is the ratio between the area of the hard region and the nominal area of the cell) and the new call arrival rate in the soft region $2^{*}\left(1-p^{\prime}\right) \lambda_{n}$, or $(1-p) \lambda_{n}$. ( $p$ is considered since it considers the overlapped area), respectively.

4) Handoff call arrival process to every cell is also considered to be a Poisson process with mean arrival rate $\lambda_{h l^{6}}$.

5) Inter-cell handoff arrival rate and the average number of users in each region $(E[\mathbf{H}]$ and $E[\mathbf{S}])$ are iteratively calculated. The inter-cell handoff arrival rate is calculated using the methodology described in (Lin et al., 1994). On the other hand, the average number of users in each region is calculated as the carried traffic in each region. This value is used to calculate interference factor in the next stage. The process stops until the carried traffic in each region converges ${ }^{7}$. This algorithm is presented in Figure 2.

6) Unencumbered service time is considered to be a negative exponentially distributed random variable with mean $1 / \mu$.

7) Residence time in each region is considered to be a negative exponentially distributed random variable with mean $1 / \eta_{x y}$, where the sub indexes $x$ and $y$ represent the current residence region and the previous residence region, respectively. When only one sub-index is used, it means that the call was originated inside the region represented by that sub-index (i.e., soft region or hard region).

8) Unencumbered call interruption time is considered to be a negative exponentially distributed random variable with mean $1 / \gamma_{x}$,

\footnotetext{
5 Since the capacity limit (i.e., maximum number of simultaneous active users in the hard and soft regions) in CDMA-based cellular systems depends on the interference level in the system, each valid state should be determined depending on the level of interference assuming that the maximum number of codes has not been allocated.

${ }^{6}$ Handoff call arrival process generated by a single cell is clearly not Poisson. However, the combined process from the six different neighboring cells can be adequately approximated by a Poisson process (Cheblus \& Ludwin, 1995).

7 Valid state space depends on the values of $E[\mathbf{S}]$ y $E[\mathbf{H}]$ and they should be calculated iteratively.
} 


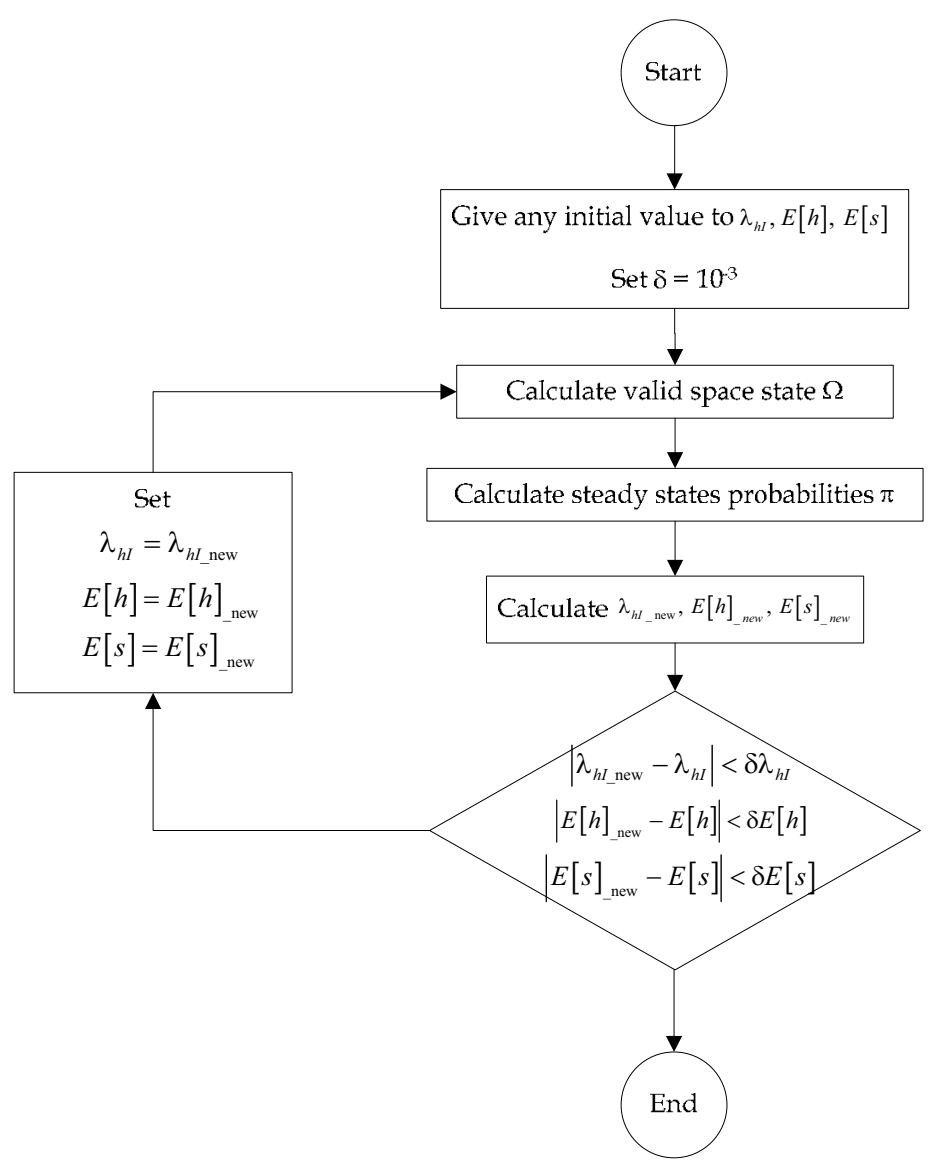

Fig. 2. Algorithm used to calculate valid space state

\subsection{Valid space state}

In order to analyze CDMA-based cellular systems considering link unreliability and soft handoff process by means of teletraffic analysis, a multi dimensional queuing analysis is necessary. Valid space state $(\Omega)$ is determined by the total number of users in the soft region $s$, and the total number of users in the hard region, $h$, given by the pairs $(s, h)$ such that a given interference constraint is met. Pairs $(s, h)$ which accomplish interference constraint depend on the system's characteristics such as processing gain $(G)$ and interference margin $(\phi)$. Consequently, if $\mathbf{Z}$ is modelled as a Gaussian random variable and considering $s$ and $h$ users in the soft and hard regions, respectively, of the analyzed cell, the probability $P_{\text {out }}(s, h)$ that $\mathbf{Z}$ exceeds a given maximum acceptable threshold is given by: 


$$
P_{\text {out }}(s, h)=\frac{1}{2}-\frac{1}{2} \operatorname{erf}\left(\frac{G(1-\phi)-E[Z(s, h)]}{\sqrt{2 \operatorname{var}[Z(s, h)]}}\right)
$$

As a result, a state is valid if it meets the condition $P_{\text {out }}(s, h) \leq P_{\text {out_max }}$ (where $P_{\text {out_max }}$ is the maximum allowed outage probability). Hence, for a given number $h$ of users in the hard region a maximum number $M_{s}(h)$ of users in the soft region can exist, as shown in Figure 3. The values of $M_{s}(h)$ represent a hard capacity limit to guarantee an acceptable performance operation in terms of the outage probability.

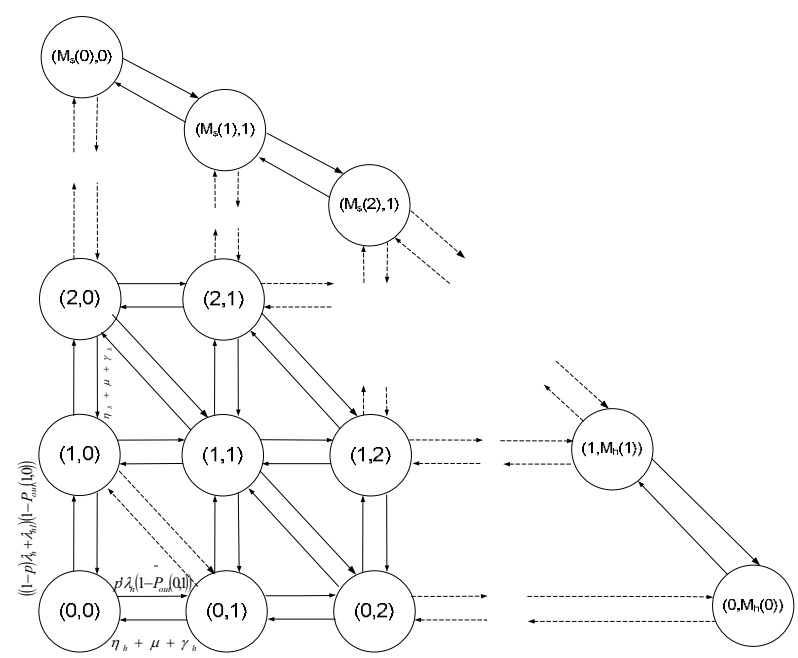

Fig. 3. Valid state space.

\subsection{Call admission control strategy}

The call admission strategy used works as follows:

When a new call or handoff attempt arrives at a given cell, the cell will try to serve the incoming request. Whether the call is accepted or not depends on the state of the system.

- If the new call is generated in the soft region or if it is an inter-cell handoff request, upon the call arrival, the condition $s+1 \leq M_{s}(h)$ is evaluated. If this condition is met, the request is accepted; otherwise, the call is blocked or dropped.

- If the new call request is generated in the hard region; upon the call arrival, the condition $s \leq M_{s}(h+1)$ is evaluated. If this condition is met, the request is accepted; otherwise, the call is blocked.

- If the request is performed by a user moving from the soft region to the hard region (intra-cell handoff), upon the request, the condition $s-1 \leq M_{s}(h+1)$ is evaluated. If this condition is met, the request is accepted; otherwise, the call is dropped. 
If the request is performed by a user coming from the hard region to the soft region (intracell handoff), upon the call arrival, the condition $s+1 \leq M_{s}(h-1)$ is evaluated. If this condition is met, the request is accepted; otherwise, the call is dropped.

\subsection{Queuing formulation}

Since a homogenous case is assumed where all cells are statistically identical, the overall system performance can be analyzed by focusing on only one given cell. Let us denote the state of a given cell as $\mathbf{K}=\left[k_{H}, k_{S} k_{S H}, k_{S S}\right]$, where $k_{H}$ and $k_{S}$ represent, respectively, the number of active users in hard and soft regions that were originated in the hard and soft regions, respectively; $k_{S H}$ represents the number of active users in the soft region of the cell coming from the hard region of the same cell, and $k_{S S}$ represents the number of active users in the soft region of the cell coming from the soft region of other cell. Let us define $\mathbf{e}_{i}$ as a vector of the same dimension of $\mathbf{K}$ whose entries are all 0 except the $i$-th one which is 1 . Then, equating rate out to rate in for each state, the statistical-equilibrium state equations are (Cooper, 1990):

$$
P_{\mathbf{K}}=\frac{\left[\sum_{i=1}^{3} a_{i \mathbf{K}-\mathbf{e}_{i}} P_{\mathbf{K}-\mathbf{e}_{i}}+\sum_{i=1}^{4} b_{i \mathbf{K}+\mathbf{e}_{i}} P_{\mathbf{K}+\mathbf{e}_{i}}+c_{1 \mathbf{K}+\mathbf{e}_{1}-\mathbf{e}_{3}} P_{\mathbf{K}+\mathbf{e}_{1}-\mathbf{e}_{3}}+\sum_{i=2}^{4} c_{i \mathbf{K}+\mathbf{e}_{i}-\mathbf{e}_{1}} P_{\mathbf{K}+\mathbf{e}_{i}-\mathbf{e}_{1}}\right]}{\left[\sum_{i=1}^{3} a_{i \mathbf{K}}+\sum_{i=1}^{4}\left(b_{i \mathbf{K}}+c_{i \mathbf{K}}\right)\right]}
$$

For $\left[k_{H}, k_{S}, k_{S H}, k_{S S}\right]$ such that $\mathrm{K} \in \Omega$.

\subsection{Transition rates}

In order to simplify the notation, let us define $s=k_{S}+k_{S S}+k_{S H}$.

The call arrival rate for users in hard region that will generate a transition from $\mathbf{K}$ to $\mathbf{K}+\mathbf{e}_{1}$ is given by the product of the mean arrival rate of the new call arrival process weighted by the probability that a user is originated in the inner region and the probability that this arrival will not exceed outage probability:

$$
a_{1 \mathbf{K}}= \begin{cases}p^{\prime} \lambda_{n}\left(1-P_{\text {out }}\left(s, k_{H}+1\right)\right) & ; s \leq M_{s}\left(k_{H}+1\right) \\ 0 & ; \text { otherwise }\end{cases}
$$

The call arrival rate for users in soft region that will generate a transition from $\mathbf{K}$ to $\mathbf{K}+\mathbf{e}_{2}$ is given by the product of the mean arrival rate of the new call arrival process weighted by the probability that a user is originated in the outer region and the probability that this arrival will not exceed outage probability: 


$$
a_{2 \mathbf{K}}= \begin{cases}(1-p) \lambda_{n}\left(1-P_{\text {out }}\left(s+1, k_{H}\right)\right) & ;(s+1) \leq M_{s}\left(k_{H}\right) \\ 0 & ; \text { otherwise }\end{cases}
$$

The call arrival rate for users in soft region that will generate a transition from $\mathbf{K}$ to $\mathbf{K}+\mathbf{e}_{4}$ is given by the product of the mean arrival rate of the handoff call arrival process and the probability that this arrival will not exceed outage probability:

$$
a_{3 \mathbf{K}}= \begin{cases}\lambda_{h I}\left(1-P_{\text {out }}\left(s+1, k_{H}\right)\right) & ;(s+1) \leq M_{s}\left(k_{H}\right) \\ 0 & ; \text { otherwise }\end{cases}
$$

The call departure rate for users in hard region that will generate a transition from $\mathbf{K}$ to $\mathbf{K}-\mathbf{e}_{1}$ is given by the sum of the mean departure rate of successfully terminated calls $(\mu)$, the mean departure rateof calls forced to terminate due to the link unreliability $\left(\gamma_{H}\right)$, and the mean departure rate of calls that are handed off to the outer region $\left(\eta_{H}\right)$, weighted by the probability that this handoff will not exceed outage probability

$$
b_{1 \mathbf{K}}= \begin{cases}k_{H}\left[\eta_{H}\left(1-P_{\text {out }}\left(s+1, k_{H}-1\right)\right)+\gamma_{H}+\mu\right] & ;(s+1) \leq M_{s}\left(k_{H}-1\right) \\ k_{H}\left[\mu+\eta_{H}+\gamma_{H}\right] & ;\left(s+1, k_{H}-1\right) \notin \Omega \\ 0 & ; \text { otherwise }\end{cases}
$$

The call departure rate of users in soft region that were originated in that region and will generate a transition from $\mathbf{K}$ to $\mathbf{K}-\mathbf{e}_{2}, \mathbf{K}-\mathbf{e}_{3}$ or $\mathbf{K}-\mathbf{e}_{4}$ is given by the sum of the mean departure rate of successfully terminated calls $(\mu)$, the mean departure rate of calls forced to terminate due to the link unreliability in the outer region $\left(\gamma_{S}\right)$, and the mean departure rate of calls that are handed off to the inner region $\left(q_{S x} \eta_{S x}\right)$, weighted by the probability that this handoff will not exceed outage probability

$$
b_{i \mathbf{K}}= \begin{cases}k_{S x}\left[\mu+\eta_{S x}\left(\left(1-q_{S x}\right)\left(1-P_{\text {out }}\left(s-1, k_{H}+1\right)\right)\right)+\gamma_{S}\right] & ;(s-1) \leq M_{s}\left(k_{H}+1\right) \\ k_{S x}\left[\mu+\eta_{S x}+\gamma_{S}\right] & ;\left(s-1, k_{H}+1\right) \notin \Omega \\ 0 & ; \text { otherwise }\end{cases}
$$

with $i=2,3,4$ and $x=\{S, S H, S S\}$ for each case.

The call departure rate for users in hard region to soft region that will generate a transition from $\mathbf{K}$ to $\mathbf{K}-\mathbf{e}_{1}+\mathbf{e}_{2}$ is given by the mean departure rate of calls that are handed off to the outer region $\left(\eta_{H}\right)$ weighted by the probability that this handoff will not exceed outage probability: 


$$
c_{1 \mathbf{K}}= \begin{cases}k_{H}\left[\eta_{H}\left(1-P_{\text {out }}\left(s+1, k_{H}-1\right)\right)\right] & ;(s+1) \leq M_{s}\left(k_{H}-1\right) \\ 0 & ; \text { otherwise }\end{cases}
$$

The call departure rate for users in soft region to hard region that will generate a transition from $\mathbf{K}$ to $\mathbf{K}-\mathbf{e}_{2}+\mathbf{e}_{1}, \mathbf{K}-\mathbf{e}_{3}+\mathbf{e}_{1}$, or $\mathbf{K}-\mathbf{e}_{4}+\mathbf{e}_{1}$ is given by the mean departure rate of calls that are handed off to the inner region $\left(q_{S x} \eta_{S x}\right)$ weighted by the probability that this handoff will not exceed outage probability:

$$
c_{i \mathbf{K}}= \begin{cases}k_{S x}\left[\eta_{S x} q_{S x}\left(1-P_{\text {out }}\left(s-1, k_{H}+1\right)\right)\right] & ;(s-1) \leq M_{s}\left(k_{H}+1\right) \\ 0 & ; \text { otherwise }\end{cases}
$$

with $i=2,3,4$, and $x=\{S, S H, S S\}$ for each case.

In addition, of course, the probabilities must satisfy the normalization equation given by (18).

$$
\sum_{k_{H}} \sum_{\substack{k_{S} \\\{\mathbf{K} \in \Omega\}}} \sum_{k_{S H}} \sum_{k_{S S}} P_{\mathbf{K}}=1
$$

The corresponding steady state probabilities are calculated by means of the Gauss-Seidel method (Cooper, 1990) by using the fixed point iteration described in Figure 2.

\subsection{QoS performance metrics (KPIs)}

The merit of this section is to derive general expressions for the QoS metrics used in this study to evaluate the performance of a CDMA-based cellular system considering both resource insufficiency and wireless link unreliability.

\subsubsection{Blocking probability}

Overall blocking probability is the weighted sum of the blocking probability of each region. New calls in soft region are blocked only if they find both cells in a blocking condition. Then, the overall blocking probability is given by (Zhang \& Lea, 2006):

$$
P_{b}=p P_{b H}+(1-p) P_{b S}^{2}
$$

Note that the probability that a call is blocked to avoid unacceptable interference (interference-limited) in every state $\left(k_{H}, k_{S}, k_{S H}, k_{S S}\right)$, should be calculated by evaluating the outage probability considering that the call was accepted. For instance, if the new call blocking probability in soft handoff region is evaluated, then outage probability should be evaluated considering that $s=k_{S}+k_{S H}+k_{S S}+1$, and $k_{H}$. In general, the following cases can be distinguished. 
- New calls in hard region could be blocked to avoid unacceptable interference (interferencelimited capacity) in every state $\left(k_{H}, k_{S}, k_{S H}, k_{S S}\right)$, with probability $P_{\text {out }}\left(s, k_{H}+1\right)$ even though the condition $s<M_{S}\left(k_{H}+1\right)$ is met. Additionally, because of resource insufficiency (hard-limited capacity), new calls in hard region are also blocked in the states where $s=M_{S}\left(k_{H}+1\right)$. Then, new call blocking probability for users originated in hard region is given by:

$$
P_{b H}=\sum_{\substack{k_{H} \\\left\{s<M s\left(k_{H}+1\right)\right\}}} \sum_{k_{S}} \sum_{k_{S S}} P_{\mathbf{K}} P_{\text {out }}\left(s, k_{H}+1\right)+\sum_{\substack{k_{H} \\\left\{s=M s\left(k_{H}+1\right)\right\}}} \sum_{k_{S}} \sum_{k_{S H}} \sum_{k_{S S}} P_{\mathbf{K}}
$$

- New calls in soft region as well as inter-cell handoff attempts could be blocked to avoid unacceptable interference (interference-limited) in every state $\left(k_{H}, k_{S}, k_{S H}, k_{S S}\right)$, with probability $P_{\text {out }}\left(s+1, k_{H}\right)$, even though the condition $(s+1)<M_{S}\left(k_{H}\right)$ is met. Additionally, due to resource insufficiency (hard-limited capacity), these calls are blocked in the states that meet the condition $(s+1)=M_{S}\left(k_{H}\right)$. Then, new call blocking probability for users originated in soft region is given by:

$$
P_{b S}=\sum_{k_{H}} \sum_{k_{S}} \sum_{k_{S H}} \sum_{\substack{k_{S S} \\\left\{(s+1)<M S\left(k_{H}\right)\right\}}} P_{\mathbf{K}} P_{\text {out }}\left(s+1, k_{H}\right)+\sum_{\substack{k_{H} \\\left\{(s+1)=M s\left(k_{H}\right)\right\}}} \sum_{k_{S H}} \sum_{k_{S S}} P_{\mathbf{K}}
$$

\subsubsection{Intracell handoff rate}

Intra-cell handoff attempt rates from hard region to soft one and from soft region to hard one (i.e., $\lambda_{h i s}$ and $\lambda_{h i H}$ ) are, respectively, calculated as the weighted sum of the handoff rate in each state multiplied by the probability to be in each state.

$$
\begin{gathered}
\lambda_{h i S}=\sum_{k_{H}} \sum_{\substack{k_{S} \\
\{\mathbf{K} \in \Omega\}}} \sum_{k_{S H}} \sum_{k_{S S}} k_{H} \eta_{H} P_{\mathbf{K}} \\
\lambda_{h I}=\sum_{k_{H}} \sum_{k_{S}} \sum_{\substack{k_{S H} \\
\{\mathbf{K} \in \Omega\}}} \sum_{k_{S S}}\left(k_{S} \eta_{S}\left(1-q_{S}\right)+k_{S H} \eta_{S H}\left(1-q_{S H}\right)+k_{S S} \eta_{S S}\left(1-q_{S S}\right)\right) P_{\mathbf{K}}
\end{gathered}
$$

\subsubsection{Intracell handoff failure probability}

Intra-cell handoff failure probability (in both directions: from hard region to soft region and vice versa) is given by the ratio of the intra-cell handoff failure rate to the intra-cell handoff attempt rate. Intra-cell handoff attempt rate is calculated as the weighted sum of the handoff rate in each state multiplied by the corresponding state probability considering only the states where handoff could be blocked. The Intra-cell handoff failure probability for subscribers moving from hard to soft regions (denoted by $F_{h i s}$ ) and for subscribers moving from soft to hard regions denoted by $F_{h i H}$ ) are given by (22) and (23), respectively. 


$$
\begin{gathered}
F_{h i H}=\sum_{k_{H}} \sum_{\substack{k_{S} \\
\left\{(s+1) \leq M_{s}\left(k_{H}-1\right)\right\}}} \sum_{k_{S S}} k_{H} \eta_{H} P_{\mathbf{K}} P_{\text {out }}\left(s+1, k_{H}-1\right) \\
F_{h i S}=\sum_{k_{H}} \sum_{\substack{k_{S} \\
\left\{(s-1) \leq M_{S} M_{S}\left(k_{H}+1\right)\right\}}} \sum_{k_{S S}}\left(k_{S} \eta_{S} q_{S}+k_{S H} \eta_{S H} q_{S H}+k_{S S} \eta_{S S} q_{S S}\right) P_{\mathbf{K}} P_{\text {out }}\left(s-1, k_{H}+1\right)
\end{gathered}
$$

Then, intra-cell handoff failure probability $P_{h i H}\left(P_{h i s}\right)$ from hard (soft) region to soft (hard) region is given by (26) and (27), respectively:

$$
\begin{gathered}
P_{h i H}=\frac{F_{h i H}}{\lambda_{h i H}} \\
P_{h i S}=\frac{F_{h i S}}{\lambda_{h i S}}
\end{gathered}
$$

\subsubsection{Interruption probability due to link unreliability}

Link unreliability is the other fundamental cause of forced termination. Considering the proposed model, a call will be forced terminated due to the link unreliability in a given region $x$ of a cell $j$ when unencumbered call interruption time is smaller than both unencumbered service time and cell dwell time. Then, mathematically, it can be expressed as:

$$
\beta_{x y}^{(j)}=P\left\{\mathbf{X}_{i_{-} x y}^{(j)}<\min \left(\mathbf{X}_{s}^{(j)}, \mathbf{X}_{r_{-} x y}^{(j)}\right)\right\} ; j=0,1,2, \ldots
$$

Due to the lack of memory of the negative exponential probability distribution of the unencumbered interruption time, unencumbered service time and cell dwell time, probability of interruption in region $x$ is given by:

$$
\beta_{x y}=\frac{\gamma_{x}}{\mu+\eta_{x y}+\gamma_{x}}
$$

As for the residence time, it is necessary to distinguish new users in the region $x$ and users handed off from region y to region $x$. Sub indexes $x$ and y represent the actual residence region and the previous residence region, respectively. 


\subsubsection{Probability of successfully call termination}

Considering the proposed model, a call will be successfully terminated in a given region $x$ of a cell $j$ when service time is smaller than both unencumbered call interruption time and cell dwell time. Then, mathematically, it can be expressed as:

$$
\zeta_{x y}^{(j)}=P\left\{\mathbf{X}_{s}^{(j)}<\min \left(\mathbf{X}_{i_{-} x y}^{(j)}, \mathbf{X}_{r_{-} x y}^{(j)}\right)\right\} ; j=0,1,2, \ldots
$$

Due to the lack of memory of the negative exponential probability distribution of the unencumbered interruption time, unencumbered service time and cell dwell time, probability of interruption in region $x$ is given by:

$$
\zeta_{x y}=\frac{\mu}{\mu+\eta_{x y}+\gamma_{x}}
$$

As for the residence time, it is necessary to distinguish new users in the region $x$ and users handed off from region $\mathrm{y}$ to region $\mathrm{x}$. Sub indexes $x$ and $\mathrm{y}$ represent the actual residence region and the previous residence region, respectively.

\subsubsection{Call forced termination probability}

Call forced termination may result from intra-cell or inter-cell handoff failure or wireless channel unreliability. A signal flow diagram is used to describe the process of a call and the call forced termination probability is obtained by using Mason's Formula (Robichaud et al., 1962). Call forced termination probability is derived by means of graph theory and Mason's rule as a mathematical tool. The steps necessary to find the forced termination probability are listed below:

- Construct a graph, where each node of this graph represents one possible state of the call. Possible states for a call are:

\section{I: Call initiation.}

$H$ : New call originated in hard region.

$S$ : New call originated in soft region.

SH: Intracellular handed off call in soft region.

SS: Intercellular handed off call in soft region

$H S$ : Intracellular handed off call in hard region

ST: Successfully terminated call.

FT: Forced terminated call.

- Connect each pair of nodes with a directed branch. Each connection is directed from the source point to the sink one. The weight of the connection is the transition probability between the states. The complete flux graph for this case is shown in Figure 4

As an illustrative example, let us assume that the weight between the ' $S$ ' node and the ' $F T$ ' node represents the probability that a call which was originated in soft region is forced to terminate. This probability is the probability that a call requires a handoff (i.e., the 
probability that residence time be less than the minimum of unencumbered service time and unencumbered interruption time which is represented by $\theta_{S}$ ). The call performs an intracellular handoff with probability $q_{S}$ and an intercellular handoff with probability $\left(1-q_{S}\right)$. An intracellular handoff will fail from soft region to hard region occurs with probability $P_{h i s}$ and an intercellular handoff will fail with probability and $P_{b s}$. Additionally, the other cause of force termination is link unreliability, (i.e., the probability that unencumbered interruption time is less than the minimum of unencumbered service time and residence time which is represented by $\beta_{S}$ ) and it occurs with probability $\beta_{S}$. Then, taking into account all of these different cases, the whole probability can be computed as $\theta_{S}\left[\left(1-q_{S}\right) P_{h I}+\left(1-q_{S}\right)\right.$ $\left.P_{b s}\right]+\beta_{s}$.

In order to obtain forced termination probability it is necessary to solve the graph by using the Mason's rule considering I as the source node and FT as the sink node (Robichaud et al., 1962). Final expression is not presented because it is very large.

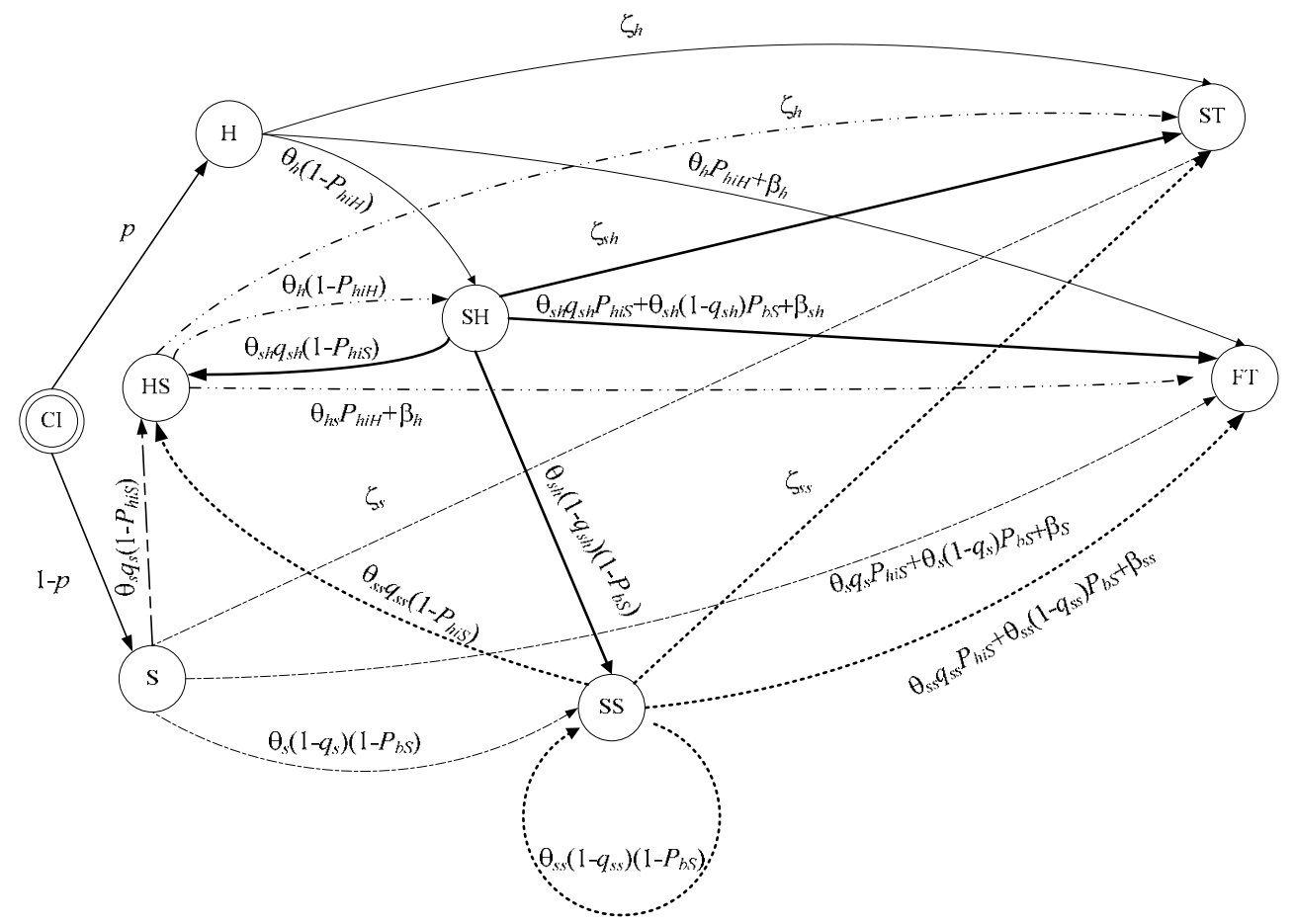

Fig. 4. Flux diagram for a call in a CDMA cellular system considering soft handoff characteristic.

\section{Performance evaluation}

The goal of the numerical evaluations presented in this section is to clarify, understand, and analyze the influence of link unreliability, user mobility, interference, soft capacity and soft handoff process on the performance metrics of CDMA-based cellular networks. 
In numerical evaluations, it is assumed that $p=0.7$, consequently, $p^{\prime}=0.85$. Mean service time is $1 / \mu=180 \mathrm{~s}$ and mean dwell time in the different regions is calculated by simulation using the mobility model of (Zoonozi \& Dassanayake, 1997) using minimum and maximum values for parameter $\alpha$ established in [38], that is, $\alpha=0^{\circ}$ (linear movement) and $\alpha=40^{\circ}$. Circular shaped cells with radius $2 \mathrm{~km}$ are considered. Mean values of these times are presented in Table 1.

\begin{tabular}{|l|l|l|}
\hline $\begin{array}{l}\text { Mobility scenario/ } \\
\text { Parameter }\end{array}$ & $\alpha=0^{\circ}$ & $\alpha=40^{\circ}$ \\
\hline $1 / \eta_{H}$ & $170 \mathrm{~s}$ & $554 \mathrm{~s}$ \\
\hline $1 / \eta_{S}$ & $46 \mathrm{~s}$ & $60 \mathrm{~s}$ \\
\hline $1 / \eta_{S S}$ & $55 \mathrm{~s}$ & $130 \mathrm{~s}$ \\
\hline $1 / \eta_{S H}$ & $50 \mathrm{~s}$ & $120 \mathrm{~s}$ \\
\hline$q_{S}$ & 0.37052 & 0.46574 \\
\hline$q_{S S}$ & 0.62902 & 0.25394 \\
\hline$q_{S H}$ & 0 & 0.64470 \\
\hline
\end{tabular}

Table 1. Users mobility parameters

The specific value of the mean unencumbered call interruption time is not easy to calculate because it closely depends on the channel and propagation conditions and the specific fading mitigation techniques. Thus, the impact of different values of mean unencumbered call interruption time are evaluated.

As in (Zhang \& Lea, 2006), considered values of interference parameters for hard and soft regions are: $m_{h}=7 \mathrm{~dB}, \sigma_{h}=\sigma_{s}=2.5 \mathrm{~dB}$. Additionally, it is assumed that the standard deviation of shadowing $\sigma=8 \mathrm{~dB}$, a processing gain $G=32$, and an interference threshold $\phi=$ $10 \mathrm{~dB}$, an offered load is $a=2$ Erlangs. Notice that the required energy per bit in soft region $m_{s}$ is a random quantity because it closely depends on the macro-diversity gain of the soft handoff process.

In addition, there are different algorithms used to implement soft handoff, thus, diversity gain depends on the specific algorithm used for soft handoff (Da Silva \& Farmine, 2005). Consequently in order to analyze the impact of this parameter on the system performance its value is varied in the range from $3.5 \mathrm{~dB}$ to $7 \mathrm{~dB}$.

Figs. 5 and 6 plot, respectively, new call blocking probability and forced termination probability versus the inverse of the mean value of the unencumbered call interruption time in hard and soft regions for two different mobility scenarios $\left(\alpha=0^{\circ}\right.$ and $\left.\alpha=40^{\circ}\right)$. Figures 7 and 8 show the global new call blocking probability and call forced termination probability versus the required bit energy to spectral interference density ratio for users in the soft region for two different users mobility scenarios $\left(\alpha=0^{\circ}\right.$ and $\left.\alpha=40^{\circ}\right)$. 


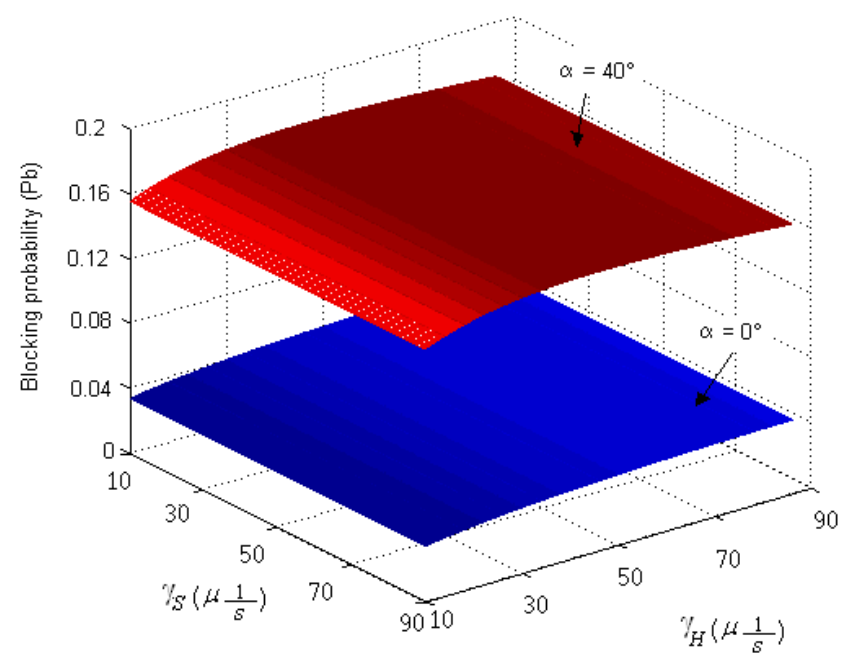

Fig. 5. New call blocking probability versus the inverse of the mean value of the unencumbered call interruption time in both regions for $\alpha=0^{\circ}$ and $\alpha=40^{\circ}$.

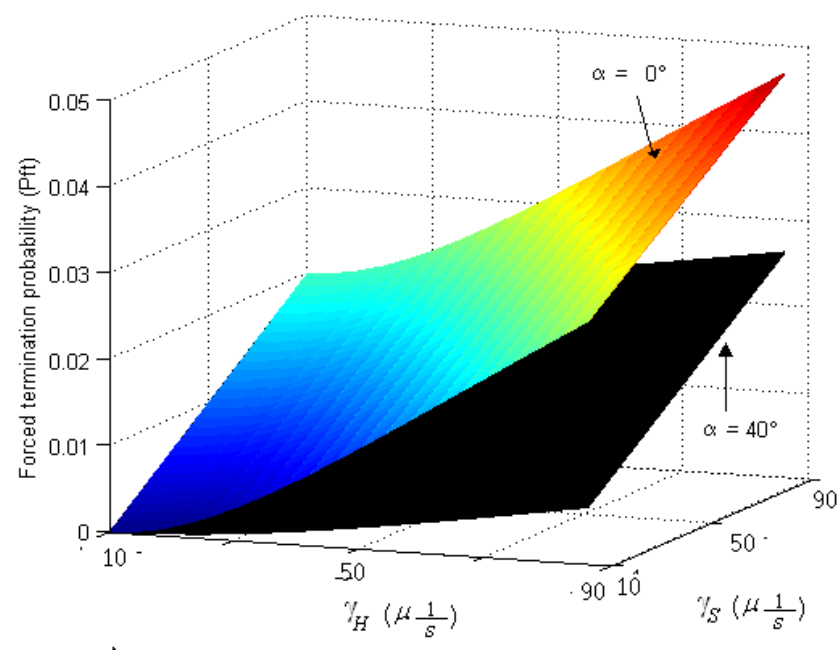

Fig. 6. Call forced termination probability versus the inverse of the mean value of the unencumbered call interruption time in both regions for $\alpha=0^{\circ}$ and $\alpha=40^{\circ}$. 


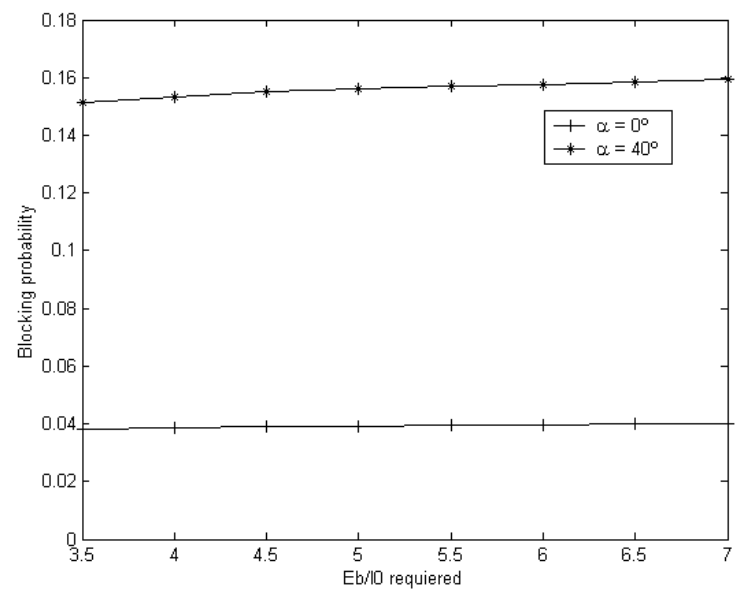

Fig. 7. New call blocking probability vs. mean value of the average requiered $E_{b} / I_{0}$ in soft handoff region $m_{s}$ for $\alpha=0^{\circ}$ and $\alpha=40^{\circ}$.

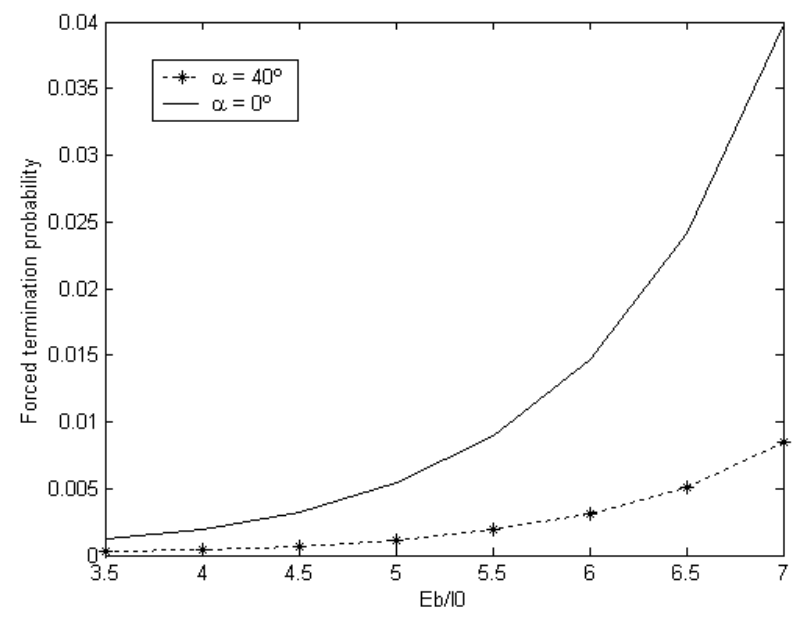

Fig. 8. Call forced termination probability vs. average requiered $\mathrm{E}_{\mathrm{b}} / \mathrm{I}_{0}$ in soft handoff region $m_{s}$ for $\alpha=0^{\circ}$ and $\alpha=40^{\circ}$.

\subsection{Influence of link unreliability}

As shown in Figure 5, new call blocking probability does not significantly vary with changes in the mean value of the unencumbered call interruption time in both regions. Nevertheless, it is observed that blocking probability increases as link unreliability in hard zones increases 
(i.e. when mean unencumbered call interruption time decreases). The call forced termination probability showed in Figure 6 has similar behavior. That is, the call forced termination probability increases as the link unreliability increases. However, the forced termination probability is more sensitive than blocking probability to changes in the mean value of the unencumbered call interruption time in both regions. This is because the interruption probability increases as link unreliability increases, directly affecting the call forced termination probability while the blocking probability remains unaffected because link unreliability is not considered to accept the call.

Call forced termination probability is the most affected QoS metric due link unreliability and also due to the soft handoff diversity. Thus, it is fundamental to consider link unreliability in the performance evaluation of CDMA-based cellular systems. Additionally, different resource allocation mechanisms designed in order to have reliable connections in CDMA-based systems have a great impact on the performance of these systems. In this work no specific strategies are considered because under the analytical framework developed in this study, the use of those reallocation mechanisms would be reflected in the reduction of the parameter $\gamma_{x}$ of the proposed call interruption process and the energy per bit requirement in soft handoff region. Then, the mathematical tool proposed here is a general instrument that can be used to evaluate the impact of new resource allocation mechanisms by considering the main features in CDMA-based cellular systems.

\subsection{Influence of mobility}

Relative to the users' mobility: the blocking probability increases as the relative users' mobility decreases (or, equivalently, as mean cell dwell time increases). This is because the larger the mean cell dwell time the slower users with ongoing calls move and the rate at which resources are released, hence, decreases. Relative to the call forced termination probability, it increases as mobility increase because more handoff arrivals are generated and is reflected directly in call forced termination probability.

Observe that blocking probability is more sensitive to changes in link unreliability in case of $\alpha=40^{\circ}$. This is because in this particular scenario (i.e.: $\alpha=40^{\circ}$ ), the mean cell dwell time is greater than $\alpha=0^{\circ}$, thus a small number of handoffs are generated, and the blocking probability is affected mainly by interruption process. The opposite effect occurs with call forced termination, because the handoff failure probability closely depends on the handoff rate and handoff failure probability.

\subsection{Influence of interference characteristics}

From the presented figures it is observed that both QoS metrics are monotonically increasing functions of the required bit energy to spectral interference density ratio. This is because the higher the offered traffic the more interference is brought into the system. Since CDMA-based systems are interference limited, more interference translates into a higher outage probability in each state, therefore, fewer users can be accepted in the system and blocking probility increases. 


\section{Conclusion}

A new system level mathematical performance analysis of mobile cellular CDMA networks was presented. Mathematical analysis takes into account main features of CDMA-based systems. Wireless channel unreliability was modeled by means of a Poisson call interruption process which allows an elegant teletraffic analysis considering both wireless link unreliability and resource insufficiency. Interference-limited capacity nature of CDMA systems was included in the teletraffic model as well as soft handoff capability considering their statistical parameters. Additionally, realistic users' movement was considered by characterizing users' mobility through the statistics of a smooth random mobility model and assuming that the user's movement is correlated.

More importantly, novel mathematical expressions for some significant performance metrics (i.e., new call blocking and call forced termination probabilities, and intra-cell and inter-cell handoff attempts rates) were derived. Graph theory and the Mason's formula were used as mathematical tools to derive a general expression for the call forced termination probability which includes both causes of forced termination: lack of resources due to the interference and wireless channel unreliability. Moreover, numerical results show that QoS metrics are evidently degraded when considering link unreliability. This results call again for the necessity of reexamining classical analytical results in traffic theory, which are used for the analysis and design of CDMA mobile networks that have not yet considered link unreliability.

On the other hand, in this work all involved times in the model were considered to be negative exponentially distributed random variables as a first step. However, future work includes the generalization of the mathematical model in order to consider general distribution for the involved time variables modelled as random variables with phase-type distributions. All these issues are subject of further future research.

\section{References}

Boggia, G.; Camarda, P.; D’Alconzo, A.; De Biasi, A.; \& Siviero, M. (2005). Drop call probability in established cellular networks: from data analysis to modelling, Proceedings of IEEE VTC'05-Spring, pp. 2775-2779, Stockholm, Sweden, May-Jun. 2005.

Chlebus, E. \& Ludwin, W. (1995). Is handoff traffic really Poissonian? Proceedings of IEEE ICUPC'95, pp. 348-353, Tokyo, Japan, November 1995.

Cooper, R.B. (1990). Introduction to Queuing Theory. CEE Press Books, Washington, D.C.

Da Silva, M. \& Farmine, Y. W-CDMA uplink soft handover gain measurements. Proceedings of IEEE VTC'05-Spring, pp. 416-418, Stockolm, Sweden, June 2005.

Dahlman, E.; Parkvall, H.; Skold J. \& Beming P. (2007). 3G Evolution. Elsevier LTD. 2007.

Elshabrawy, T. \& Le-Ngoc, T. (2005). Gilbert channel approximation for downlink performance evaluation of WCDMA systems. Proceedings of IEEE VTC'05-Fall, pp. 382-386, Dallas, TX, September 2005.

Garg V. (2000). IS-95CDMA and cdma2000: cellular/PCS implementation. Prentice Hall, New York.

Hegde, N. \& Sohraby, K. (2002). On the impact of soft handoff in cellular systems, Computer Networks, Vol. 38, No.2, February 2002, pp. 257-271.

Kim D.K. \& Sung, D.K. (1999). Characterization of soft handoff in CDMA systems. IEEE Transactions on Vehicular Technology, Vol. 48, No. 4, July 1999, pp.1195-1202. 
Kong, P. Y. (2002). Performance of queue in impaired wireless channel. IEE Electronics Letters, Vol. 38, No. 22, October 2002, pp. 1342-1343.

Li, B.; Li, L.; Li, B.; Sivalingam, K.M. \& Cao, X.R. (2004). Call admission control for voice/data integrated cellular networks: performance analysis and comparative study. IEEE Journal on Selected Areas of Communications, Vol. 22, No. 4, May 2004, pp. 706-718.

Lin, Y.B.; Mohan, S. \& Noerpel, A. (1994). Queuing priority channel assignment strategies for PCS and handoff initial access. IEEE Transactions on Vehicular Technology, Vol. 43. No. 3, August 1994, pp. 704-712.

Liu, B. \& Sule Alfa, A., (2004). A queueing model with time-varying QoS and call dropping for evaluating the performance of CDMA cellular systems, Wireless Communication and Mobile Computing, No. 4, March 2004, pp. 439-447.

Ma, X.; Liu, Y. \& Trivedi, K. S. (2006). Modeling and performance analysis for soft handoff schemes in CDMA cellular systems. IEEE Transaction on Vehicular Technology, Vol. 55, No. 2, March 2006, pp. 670-680.

Naraghi-Pour, M. \& Chai, Y. (2006). Call admission control for CDMA cellular networks supporting multimedia services. Proceedings of IEEE WCNC'06, pp. 997-1002, Las Vegas, NV, May 2006.

Piao, S.; Park, J. \& Park, Y. (2006). Outage probability analysis for DS-CDMA systems with call admission control schemes. IEICE Transactions on Communications, Vol. E89B, No. 8, August 2006, pp. 2226-2229.

Robichaud, L.P.A.; Boisvert, M. \& Robert, J. (1962). Signal Flow Graphs and Applications. Prentice Hall, New Jersey.

Rodriguez-Estrello, C.B.; Cruz-Pérez, F.A. \& Hernández-Valdez, G. (2009). System level analysis of mobile cellular networks considering link unreliability. IEEE Transactions on Vehicular Technology, Vol. 58, No. 2, , February 2009, pp. 926-940.

Su, L.; Chen, J.Y. \& Huang, J.H. (1996) Performance analysis of soft handoff in CDMA Cellular Networks. IEEE Journal on Selected Areas of Communication, Vol. 14, No. 9, December 1996, pp. 1762-1769.

Viterbi, A.J.; Viterbi, A.M. \& Zehavi, E. (1994). Other cell interference in cellular power controlled CDMA. IEEE Transactions on Communications. Vol. 42, No. 2/3/4, February/March/April 1994, pp. 1501-1504.

Wong, K.Y.M.; Keung, Y.; Li, C. \& Li, B. (2004). Dynamic call admission control with connection level service guarantee in multi-service wireless cellular networks. Proceedings of IEEE ICC'04, pp. 4248-4252, Paris, France, June 2004.

Zhang, M. \& Lea, C.T. (2006). The impact of mobility on CDMA call admission control. IEEE Transactions on Vehicular Technology, Vol. 55, No. 6, November 2006, pp. 1908-1920.

Zhang, Y. \& Soong, B. (2005). The effect of unreliable wireless channel on the call performance in mobile network. IEEE Transanctions on Wireless Communications, Vol. 4, No.2, March 2005, pp. 653-661.

Zhang, Y. \& Soong, B. (2006). Performance of mobile network with wireless channel unreliability and resource insufficiency. IEEE Transanctions on Wireless Communications, Vol. 5, No. 5, May 2006, pp. 990-995.

Zhao, D.; Shen, X. (Sherman); \& Mark, J.W. (2006). Soft handoff and connection reliability in cellular CDMA downlinks. IEEE Transactions on Wireless Communications, Vol. 5, No. 2, February 2006, pp. 354-365

Zonoozi, M.M. \& Dassanayake, P. (1997). User mobility modelling and characterization of mobility patterns. IEEE Selected Areas on Communication, Vol. 15, No. 7, September 1997, pp. 1239-1252. 


\section{Mobile and Wireless Communications Physical Layer Development and Implementatiom}

Edited by Salma Ait Fares and Fumiyuki Adachi

ISBN 978-953-307-043-8

Hard cover, 290 pages

Publisher InTech

Published online 01, January, 2010

Published in print edition January, 2010

Mobile and Wireless Communications have been one of the major revolutions of the late twentieth century. We are witnessing a very fast growth in these technologies where mobile and wireless communications have become so ubiquitous in our society and indispensable for our daily lives. The relentless demand for higher data rates with better quality of services to comply with state-of-the art applications has revolutionized the wireless communication field and led to the emergence of new technologies such as Bluetooth, WiFi, Wimax, Ultra wideband, OFDMA. Moreover, the market tendency confirms that this revolution is not ready to stop in the foreseen future. Mobile and wireless communications applications cover diverse areas including entertainment, industrialist, biomedical, medicine, safety and security, and others, which definitely are improving our daily life. Wireless communication network is a multidisciplinary field addressing different aspects raging from theoretical analysis, system architecture design, and hardware and software implementations. While different new applications are requiring higher data rates and better quality of service and prolonging the mobile battery life, new development and advanced research studies and systems and circuits designs are necessary to keep pace with the market requirements. This book covers the most advanced research and development topics in mobile and wireless communication networks. It is divided into two parts with a total of thirty-four stand-alone chapters covering various areas of wireless communications of special topics including: physical layer and network layer, access methods and scheduling, techniques and technologies, antenna and amplifier design, integrated circuit design, applications and systems. These chapters present advanced novel and cutting-edge results and development related to wireless communication offering the readers the opportunity to enrich their knowledge in specific topics as well as to explore the whole field of rapidly emerging mobile and wireless networks. We hope that this book will be useful for students, researchers and practitioners in their research studies.

\section{How to reference}

In order to correctly reference this scholarly work, feel free to copy and paste the following:

Carmen B. Rodriguez-Estrello, Genaro Hernandez Valdez and Felipe A. Cruz Perez (2010). Performance Modelling and Analysis of Mobile Wireless Networks, Mobile and Wireless Communications Physical Layer Development and Implementatiom, Salma Ait Fares and Fumiyuki Adachi (Ed.), ISBN: 978-953-307-043-8, InTech, Available from: http://www.intechopen.com/books/mobile-and-wireless-communications-physical-layerdevelopment-and-implementatiom/performance-modelling-and-analysis-of-mobile-wireless-networks

\section{INTECH}




\section{InTech Europe}

University Campus STeP Ri

Slavka Krautzeka 83/A

51000 Rijeka, Croatia

Phone: +385 (51) 770447

Fax: +385 (51) 686166

www.intechopen.com

\section{InTech China}

Unit 405, Office Block, Hotel Equatorial Shanghai

No.65, Yan An Road (West), Shanghai, 200040, China

中国上海市延安西路65号上海国际贵都大饭店办公楼 405 单元

Phone: +86-21-62489820

Fax: $+86-21-62489821$ 
(C) 2010 The Author(s). Licensee IntechOpen. This chapter is distributed under the terms of the Creative Commons Attribution-NonCommercial-ShareAlike-3.0 License, which permits use, distribution and reproduction for non-commercial purposes, provided the original is properly cited and derivative works building on this content are distributed under the same license. 